\title{
Beaming Effects in Gamma-Ray Burst Afterglows
}

\author{
Y. F. Huang, T. Lu, Z. G. Dai \\ Dept Astronomy, Nanjing University, Nanjing 210093, China
}

\author{
K. S. Cheng \\ Dept Physics, the University of Hong Kong, Hong Kong, China
}

\begin{abstract}
Based on a refined dynamical model, afterglows from jetted $\gamma$-ray burst (GRB) remnants are investigated numerically. Measuring of GRB beaming by using orphan afterglow surveys is addressed. The possible existence of a kind of cylindrical jets is also discussed.
\end{abstract}

\section{Introduction}

The importance of the non-relativistic phase in GRB afterglows has been stressed by Huang et al. $(1998 \mathrm{a}, \mathrm{b})$. Conventional dynamical model is not correct in the non-relativistic phase. Huang et al. (1999) have proposed a refined equation,

$$
\frac{d \gamma}{d m}=-\frac{\gamma^{2}-1}{M_{\mathrm{ej}}+\epsilon m+2(1-\epsilon) \gamma m},
$$

where $\epsilon$ is the radiative efficiency. This equation is correct in both ultrarelativistic and non-relativistic phases (c.f. Fig. 1). Very long time radio light curves have been obtained by Kulkarni et al. for some GRBs (Kulkarni 2002). Our model should be helpful in fitting the radio data. Here, we use the refined dynamical model to study the jet effect on the afterglow light curves.

\section{Beaming Effects}

Beaming effects have been discussed a lot by many authors (Kulkarni et al. 1999; Zhang \& Mészáros 2002). Figs. 3 and 4 illustrate the effect of electron energy ratio and magnetic energy ratio on the R-band light curves. When $\xi_{\mathrm{e}}$ and $\xi_{\mathrm{B}}$ are small, an obvious break does appear in the light curve, but it is clearly due to the relativistic-Newtonian transition. The effects of other parameters on the afterglows have also been discussed by Huang et al. (2000c). It is strongly suggested that the rapid fading of afterglows currently observed in some GRBs is evidence for beaming (Huang et al. 2000a, b). Afterglows from cylindrical GRB jets have also been discussed in great detail by Cheng et al. (2001).

The ratio of the orphan afterglow rate to the GRB rate might allow measurement of the GRB collimation angle. However, the problem is greatly complicated because there should exist many "failed GRBs (FGRBs)", i.e., baryoncontaminated fireballs with initial Lorentz factor $1 \ll \gamma_{0} \ll 100$ (Huang et 

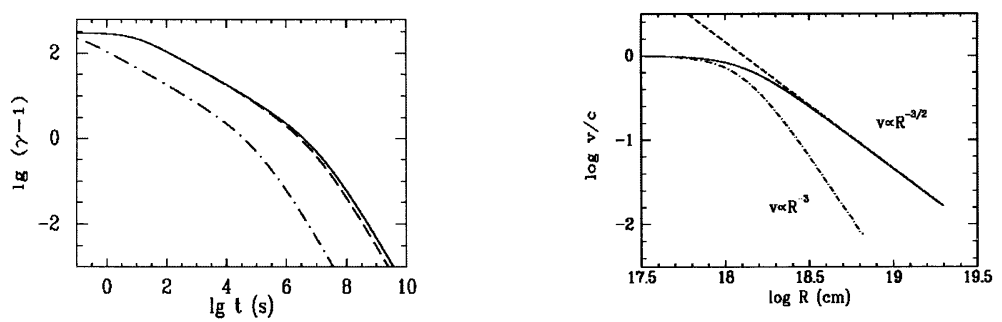

Figure 1. Left panel illustrates the evolution of $\gamma$ for some exemplary GRB jets, which becomes non-relativistic at time $10^{5}-10^{6}$ (Huang et al. 2000c). Right panel illustrates the velocity vs. radius for an adiabatic fireball (Huang 2000), where dashed line is the familiar Sedov solution in Newtonian phase and solid line corresponds to our refined model (Eq. (1)), which is consistent with the Sedov solution.
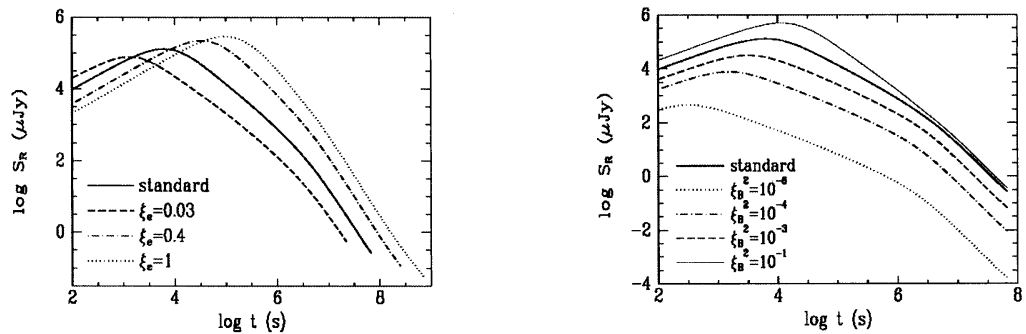

Figure 2. Effects of $\xi_{\mathrm{e}}$ and $\xi_{\mathrm{B}}$ on afterglows (Huang et al. 2000c).

al. 2002). X-ray observations can provide valuable clues to the progenitors of GRBs (Antonelli et al. 2000). The so called X-ray-GRBs recently discovered by BeppoSAX might just be FGRBs (Huang et al. 2002).

\section{References}

Antonelli, L. A., et al. 2000, ApJ, 545, L39

Cheng, K. S., Huang, Y. F., \& Lu, T. 2001, MNRAS, 325, 599

Huang, Y. F. 2000, astro-ph/0008177

Huang, Y. F., Dai, Z. G., \& Lu, T. 1998a, A\&A, 336, L69

Huang, Y. F., Dai, Z. G., \& Lu, T. 1998b, MNRAS, 298, 459

Huang, Y. F., Dai, Z. G., \& Lu, T. 1999, MNRAS, 309, 513.

Huang, Y. F., et al. 2000a, ApJ, 543, 90

Huang, Y. F., Dai, Z. G., \& Lu, T. 2000b, A\&A, 355, L43

Huang, Y. F., Dai, Z. G., \& Lu, T. 2000c, MNRAS, 316, 943

Huang, Y. F., Dai, Z. G., \& Lu, T. 2002, MNRAS, 332, 735

Kulkarni, S. R., et al., 1999, Nat, 398, 389

Kulkarni, 2002, in this volume

Zhang, B. \& Mészáros, P. 2002, ApJ, 571, 876 